\title{
Hypoalbuminemia, An Independent Risk Factor for Severity and Mortality Affects One Third of Patients in Acute Pancreatitis: Multicenter Prospective International Cohort Analysis
}

Klementina Ocskay

University of Pécs

Zsófia Vinkó

University of Pécs

Dávid Németh

University of Pécs

László Szabó

University of Pécs

Judit Bajor

University of Pécs

Szilárd Gódi

University of Pécs

Patrícia Sarlós

University of Pécs

László Czakó

University of Szeged

Ferenc Izbéki

Szent György University Teaching Hospital of Fejér County

József Hamvas

Peterfy Hospital

Mária Papp

University of Debrecen

Márta Varga

Dr. Réthy Pál Hospital

Imola Török

University of Medicine, Pharmacy, Sciences and Technology

Artautas Mickevicius

Vilnius University Hospital Santaros Clinics

Ville Sallinen

University of Helsinki 
Elena Ramirez Maldonado

Consorci Sanitari del Garraf, Sant Pere de Ribes

Shamil Galeev

Saint Luke Clinical Hospital

Alexandra Mikó

University of Pécs

Bálint Erốss

University of Pécs

Marcell Imrei

University of Pécs

Péter Jenố Hegyi

University of Pécs

Nándor Faluhelyi

University of Pécs

Orsolya Farkas

University of Pécs

Péter Kanizsai

University of Pécs

Attila Miseta

University of Pécs

Tamás Nagy

University of Pécs

Roland Hágendorn

University of Pécs

Zsolt Márton

University of Pécs

Zsolt Szakács

University of Pécs

Andrea Szentesi

University of Pécs

Péter Hegyi

University of Pécs

Andrea Párniczky ( $\square$ andrea.parniczky@gmail.com )

Heim Pál National Pediatric Institute

Hungarian Pancreatic Study Group

University of Pécs

Research Article 
Keywords: human serum albumin, complication, mortality, severity, prognostic factor, logistic regression, receiver operating curve

Posted Date: June 17th, 2021

DOI: https://doi.org/10.21203/rs.3.rs-612796/v1

License: (1) This work is licensed under a Creative Commons Attribution 4.0 International License. Read Full License 


\section{Abstract}

Introduction The incidence and medical costs of acute pancreatitis (AP) are on the rise, and severe cases still have a $30 \%$ mortality rate. We aimed to evaluate hypoalbuminemia as a risk factor and the prognostic value of human serum albumin in AP.

Methods Data of 2461 patients were extracted from the international, prospective, multicenter AP registry of the Hungarian Pancreatic Study Group. Data of patients with albumin measurement in the first 48 hours $(n=1149)$ and anytime during hospitalization $(n=1272)$ was analyzed. Multivariate binary logistic regression and Receiver Operator Characteristic curve analysis were used.

Results The prevalence of hypoalbuminemia $(<35 \mathrm{~g} / \mathrm{L})$ was $19 \%$ on-admission and $35.7 \%$ during hospitalization. Hypoalbuminemia dose-dependently increased the risk of severity, mortality, local complications, and organ failure and is associated with longer hospital stay. The predictive value of hypoalbuminemia on-admission was poor for severity and mortality. Severe hypoalbuminemia $(<25 \mathrm{~g} / \mathrm{L})$ was an independent risk factor for severity (OR: 48.761; Cl:25.276-98.908) and mortality (OR:16.83; Cl: 8.32-35.13). Albumin loss during AP was strongly associated with severity $(p<0.001)$ and mortality $(p=0.002)$.

Conclusion Hypoalbuminemia is an independent risk factor of severity and mortality in AP, and it shows a dose-dependent relationship with local complications, organ failure, and length of stay.

\section{Introduction}

Acute pancreatitis is a common gastroenterological disorder, with rising incidence and high medical costs. The commonly used revised Atlanta Classification distinguishes between mild, moderate, and severe disease by the development and duration of organ failure ${ }^{1}$. As the mortality rate can reach $30 \%$ in severe cases, identifying risk factors and potential therapeutic targets is of utmost importance.

Human serum albumin is the most abundant protein in human serum, with a very diverse role. Although this hypothesis was contradicted by recent data, declining albumin levels during inflammation for long prompted physicians to underestimate its contribution to maintaining homeostasis during inflammation. However, it plays a pivotal role in maintaining the plasma redox state ${ }^{2}$, and its scavenging activity is likely to influence vascular resistance through the regulation of nitric oxide levels ${ }^{3}$. Furthermore, low albumin levels result in dilution and increased drug clearance, ultimately causing sub-optimal treatment ${ }^{4}$.

Small retrospective cohort studies showed that hypoalbuminemia is an independent risk factor for severe AP and in-hospital mortality in adults and children ${ }^{5,6}$. Serum albumin was reported to be associated with persistent organ failure and prolonged hospital stay ${ }^{7}$. However, whether albumin is only a marker or there is a cause-effect relationship between hypoalbuminemia and disease severity and mortality should be further evaluated. 
While comprehensive analyses are missing on AP patients with hypoalbuminemia and albumin loss in $A P$, we aimed to evaluate (1) on-admission and in-hospital hypoalbuminemia as a risk factor in $A P,(2)$ the prognostic potential of human serum albumin, (3) whether there is a dose-dependent relationship between albumin level and disease outcomes and (4) the association of albumin loss with severity and mortality.

We found evidence that AP patients with $<25 \mathrm{~g} / \mathrm{L}$ serum albumin anytime during hospitalization have a 16.8 times higher risk of death and 48.8 times higher risk of severe AP than patients with normal albumin levels. We also pointed out that albumin loss during AP is associated with severity and mortality. These data highlight the unmet need for randomized controlled trials focusing on albumin replacement.

\section{Results}

\section{One in every five patients suffering from acute pancreatitis has hypoalbuminemia on admission}

Nineteen percent of patients $(n=218 / 1149)$ presented with hypoalbuminemia $(<35 \mathrm{~g} / \mathrm{L}) .12 .4 \%$ of patients were admitted with $30-34.99 \mathrm{~g} / \mathrm{L}$ albumin levels (group 5), whereas $4.4 \%$ and $2.2 \%$ of patients had 25-29.99 g/L (group 6) and < $25 \mathrm{~g} / \mathrm{L}$ (group 7) on-admission albumin levels (Supplementary Fig. S3).

Older age, lower body mass index, abdominal guarding, on physical examination and non-biliary etiology are associated with on admission hypoalbuminemia

Hypalbuminemia was associated with older age (average $59.7 \pm 18.0$ and $56.0 \pm 16.1$ years; $p=0.005$, Supplementary Fig. S3). Males were overrepresented in the analyzed cohort (57\%) and all subgroups (Fig. S3). Although biliary etiology was the most frequent in all subgroups, significantly fewer patients had biliary etiology ( $34.4 \%$ versus $42.2 \% ; p=0.042)$ in the low albumin group, and a tendency of more alcoholic episodes ( $24.3 \%$ and $19 \% ; p=0.096)$ was seen (Supplementary Fig. S3).

Significantly lower body mass index (average 28.23 and 27.23; $p=0.012$ ) was found in the low albumin group compared to the normal albumin group (Supplementary Fig. S4). Diabetes mellitus (22.6\% versus $19.3 \% ; p=0.318)$ and chronic pancreatitis $(7.3 \%$ versus $6.1 \%, p=0.507)$ were overrepresented in patients with hypoalbuminemia, however, fewer patients with hypoalbuminemia had recurrent AP $(17.4 \%$ versus $21.9 \%, p=0.144$ ) (Supplementary Fig. S4.).

Considering the signs and symptoms, fewer hypoalbuminemia patients presented with abdominal pain (94.9\% and $99.2 \% ; p<0.001)$ and more with abdominal guarding (27.2\% and $19.9 \% ; p=0.023)$ (Fig. S5). General signs, such as duration and intensity of abdominal pain, abdominal tenderness, nausea, and vomiting, did not significantly differ. Hypoalbuminemia was associated with a dose-dependent increase in heart rate and a decrease in systolic and diastolic blood pressures on admission (Supplementary Fig. S5). 
The fulfillment of diagnostic criteria differed significantly $(p<0.001)$ among the low and normal albumin groups on-admission. Low albumin patients were less likely to present with pancreatic enzyme elevation, abdominal pain, and characteristic imaging findings at the same time ( $42.7 \%$ versus $58.4 \%$ ) (Supplementary Table S2).

\section{On-admission hypoalbuminemia is dose-dependently associated with elevated CRP and PCT levels in AP}

The low albumin group had significantly lower serum amylase $(p<0.001)$ and lipase $(p=0.002)$ levels on admission. A dose-dependent C-reactive protein (CRP) $(p<0.001)$ and procalcitonin (PCT) $(p<0.001)$ increase was observed in the lower albumin groups. White blood cell count (WBC) $(p=0.017)$ levels were also significantly elevated in the low albumin group (Fig. S6-7). Concerning laboratory markers of renal function, hypoalbuminemia patients had significantly higher blood urea nitrogen (BUN) $(p=0.002)$ and creatinine $(p=0.002)$ levels and lower estimated glomerular filtration rate (eGFR) $(p<0.001)$ (Supplementary Fig. S8-9). Liver enzymes and total bilirubin levels did not differ between the low and normal albumin groups, but hypoalbuminemia was associated with higher direct bilirubin levels $(p=$ $0.005)$ and a higher international normalized ratio (INR) $(p<0.001)$ (Supplementary Fig. S10-13). Hematological parameters, lipids, ions, and glucose levels are shown in Supplementary Figures S14-17.

\section{On-admission hypoalbuminemia is dose-dependently associated with complications, severity, and mortality in AP}

Significantly more patients developed local complications, and organ failure in the low albumin group ( $p$ $=0.016$ and $p<0.001$, respectively) (Fig. 1-2). Lower albumin levels correlated with a higher rate of peripancreatic fluid collection and respiratory failure $(p<0.001$ and $p=0.051)$. The rate of pancreatic necrosis, pseudocyst, or heart failure did not differ significantly between the groups.

All types of local complications were significantly more frequent in the low albumin group. A dosedependent increase was seen in the rate of local complications and peripancreatic fluid collection in both cohorts and in pancreatic necrosis and pseudocyst in the lowest measured albumin cohort. $P<0.05$ is considered significant. Patients with albumin levels $<35 \mathrm{~g} / \mathrm{L}$ were included in the low albumin group (groups 5-7).

Significantly more patients developed organ failure in the low albumin group in both cohorts. A dosedependent increase was seen in the case of all analyses in the lowest measured albumin cohort. Heart failure was dose-dependently increased in the on-admission cohort as well. $P<0.005$ is considered significant.

Most importantly, hypoalbuminemia was associated with increased mortality $(p=0.020)$, disease severity $(p=0.015)$, and hospital stay $(p=0.025)$ (Fig. 3). Groups 6 and 7 had significantly higher mortality $(p=$ 0.005 and $p=0.007$, respectively $)$ and severity $(p=0.028$ and $p<0.001$, respectively $)$ compared to the 
normal group. Maximum CRP levels during the course of AP significantly and dose-dependently increased with the degree of serum albumin ( $p<0.001$, Fig. 3$)$.

On-admission hypoalbuminemia is an independent risk factor of severity and mortality, with an odds ratio up to 5.3 for mortality in acute pancreatitis

Age, hypertriglyceridemia-induced (with or without concomitant alcoholic etiology), and idiopathic AP were independently associated with mortality. Severe on-admission hypoalbuminemia proved to be an independent risk factor for mortality with an OR of 3.782 (Cl: 1.313-9.462) in group $6(<30 \mathrm{~g} / \mathrm{L})$ and an OR of 5.256 (Cl: 1.389-16.112) in group 7 (<25 g/L) (Table 1.) Albumin levels were examined with a 35 $\mathrm{g} / \mathrm{L}$ cut-off in a separate analysis, which found an independent association between hypoalbuminemia and mortality (OR: 2.070; Cl: 1.021-4.033; Supplementary Table S1). Age, hypertriglyceridemia-induced $\mathrm{AP}$, and, among the multifactorial etiologies the combination of hypertriglyceridemia and alcohol were independent risk factors of disease severity. On-admission albumin levels $<25 \mathrm{~g} / \mathrm{L}$ were independently associated with severe AP (OR: 3.620; Cl: 1.128-9.978; Table 1). 
Table 1

- Multivariate logistic regression analysis on the prognostic role of on-admission hypoalbuminemia in acute pancreatitis

\section{On-admission albumin $(n=1149)$ - mortality}

\begin{tabular}{|c|c|c|c|c|c|c|}
\hline \multicolumn{2}{|l|}{ Predictor } & \multirow{2}{*}{$\begin{array}{l}\beta \\
-0.108\end{array}$} & \multirow{2}{*}{$\begin{array}{l}\text { SE } \\
0.553\end{array}$} & \multirow{2}{*}{$\begin{array}{l}\text { OR } \\
0.898\end{array}$} & \multirow{2}{*}{$\begin{array}{l}95 \% \mathrm{Cl} \\
0.259- \\
2.390\end{array}$} & \multirow{2}{*}{$\begin{array}{l}p \\
0.845\end{array}$} \\
\hline $\begin{array}{l}\text { On-admission albumin } \\
\text { level }\end{array}$ & $\begin{array}{l}30-34.99 \mathrm{~g} / \mathrm{L}(\mathrm{vs} . \geq 35 \\
\mathrm{g} / \mathrm{L})\end{array}$ & & & & & \\
\hline & $\begin{array}{l}25-29.99 \mathrm{~g} / \mathrm{L}(\mathrm{vs} . \geq 35 \\
\mathrm{g} / \mathrm{L})\end{array}$ & 1.330 & 0.496 & 3.782 & $\begin{array}{l}1.313- \\
9.462\end{array}$ & 0.007 \\
\hline & $<25 \mathrm{~g} / \mathrm{L}(\mathrm{vs} . \geq 35 \mathrm{~g} / \mathrm{L})$ & 1.659 & 0.611 & 5.256 & $\begin{array}{l}1.389- \\
16.112\end{array}$ & 0.007 \\
\hline Age & per years & 0.037 & 0.012 & 1.037 & $\begin{array}{l}0.014- \\
1.063\end{array}$ & 0.003 \\
\hline Gender & female (vs. male) & -0.222 & 0.370 & 0.801 & $\begin{array}{l}0.383- \\
1.648\end{array}$ & 0.548 \\
\hline \multirow[t]{7}{*}{ Etiology } & alcohol (vs. biliary) & 0.669 & 0.554 & 1.952 & $\begin{array}{l}0.636- \\
5.725\end{array}$ & 0.227 \\
\hline & HTG (vs. biliary) & 1.669 & 0.747 & 5.304 & $\begin{array}{l}1.037- \\
21.022\end{array}$ & 0.025 \\
\hline & $\begin{array}{l}\text { biliary + alcohol (vs. } \\
\text { biliary) }\end{array}$ & 1.234 & 1.100 & 3.436 & $\begin{array}{l}0.178- \\
20.816\end{array}$ & 0.262 \\
\hline & $\begin{array}{l}\text { biliary + HTG (vs. } \\
\text { biliary) }\end{array}$ & -12.903 & 783.282 & - & - & 0.987 \\
\hline & $\begin{array}{l}\text { alcohol + HTG (vs. } \\
\text { biliary) }\end{array}$ & 1.781 & 0.768 & 5.938 & $\begin{array}{l}1.123- \\
24.693\end{array}$ & 0.020 \\
\hline & idiopathic (vs. biliary) & 1.119 & 0.427 & 3.061 & $\begin{array}{l}1.330- \\
7.223\end{array}$ & 0.009 \\
\hline & other (vs. biliary) & 0.010 & 0.790 & 1.010 & $\begin{array}{l}0.152- \\
3.964\end{array}$ & 0.990 \\
\hline \multicolumn{7}{|c|}{ On-admission albumin $(n=1149)$ - severity } \\
\hline Predictor & & $\beta$ & SE & OR & $95 \% \mathrm{Cl}$ & $\mathrm{p}$ \\
\hline \multirow[t]{3}{*}{ On-admission albumin } & $\begin{array}{l}30-34.99 \mathrm{~g} / \mathrm{L}(\mathrm{v} . \geq 35 \\
\mathrm{g} / \mathrm{L})\end{array}$ & 0.029 & 0.383 & 1.030 & $\begin{array}{l}0.457- \\
2.086\end{array}$ & 0.939 \\
\hline & $\begin{array}{l}25-29.99 \mathrm{~g} / \mathrm{L}(\mathrm{v} . \geq 35 \\
\mathrm{g} / \mathrm{L})\end{array}$ & 0.829 & 0.449 & 2.292 & $\begin{array}{l}0.882- \\
5.238\end{array}$ & 0.065 \\
\hline & $<25 \mathrm{~g} / \mathrm{L}(\mathrm{v} . \geq 35 \mathrm{~g} / \mathrm{L})$ & 1.286 & 0.548 & 3.620 & $\begin{array}{l}1.118- \\
9.968\end{array}$ & 0.019 \\
\hline
\end{tabular}




\begin{tabular}{|c|c|c|c|c|c|c|}
\hline \multicolumn{7}{|c|}{ On-admission albumin $(n=1149)$ - mortality } \\
\hline Age & per years & 0.040 & 0.010 & 1.041 & $\begin{array}{l}1.022- \\
1.061\end{array}$ & $\begin{array}{l}<.001 \\
0.001\end{array}$ \\
\hline Gender & female (vs. male) & -0.183 & 0.281 & 0.830 & $\begin{array}{l}0.478- \\
1.442\end{array}$ & 0.515 \\
\hline \multirow[t]{7}{*}{ Etiology } & alcohol (vs. biliary) & 0.522 & 0.420 & 1.685 & $\begin{array}{l}0.751- \\
3.673\end{array}$ & 0.195 \\
\hline & HTG (vs. biliary) & 1.712 & 0.546 & 5.543 & $\begin{array}{l}1.776- \\
15.536\end{array}$ & 0.002 \\
\hline & $\begin{array}{l}\text { biliary + alcohol (vs. } \\
\text { biliary) }\end{array}$ & 1.056 & 0.802 & 2.874 & $\begin{array}{l}0.426- \\
11.572\end{array}$ & 0.188 \\
\hline & $\begin{array}{l}\text { biliary + HTG (vs. } \\
\text { biliary) }\end{array}$ & -13.792 & 785.525 & - & - & 0.986 \\
\hline & $\begin{array}{l}\text { alcohol + HTG (vs. } \\
\text { biliary) }\end{array}$ & 1.316 & 0.632 & 3.727 & $\begin{array}{l}0.952- \\
11.941\end{array}$ & 0.037 \\
\hline & idiopathic (vs. biliary) & 0.536 & 0.330 & 1.709 & $\begin{array}{l}0.884- \\
3.247\end{array}$ & 0.104 \\
\hline & other (vs. biliary) & -0.475 & 0.629 & 0.622 & $\begin{array}{l}0.145- \\
1.852\end{array}$ & 0.450 \\
\hline
\end{tabular}

HTG: hypertriglyceridemia; $\beta$ : $\beta$ coefficient; SE: standard error; OR: odds ratio; Cl: confidence interval

\section{On-admission albumin levels alone have poor predictive value in AP}

On-admission albumin levels have an AUC of 0.615 (sensitivity: $57.6 \%$, specificity: $61.1 \%$ ) for the severity with a cut-off at $39.3 \mathrm{~g} / \mathrm{L}$ (Fig. 4). The AUC for mortality was 0.660 (sensitivity: $72.1 \%$, specificity: $53.7 \%$ ) with a cut-off at $37.0 \mathrm{~g} / \mathrm{L}$.

These data prompt that albumin plays a crucial role in the pathophysiology and clinical outcome of AP, however cannot be used as a single biomarker for predicting severity and mortality. Next, we wanted to understand whether albumin loss during the course of AP has any association with the outcome of the disease; therefore, we regrouped our patients based on the lowest measured albumin levels.

One out of three patients suffer from hypalbuminemia in AP during hospitalization, which dosedependently correlates with disease severity and mortality in AP

The proportion of patients with hypoalbuminemia anytime during hospitalization was $35.7 \%$ (454 patients). A significant, dose-dependent increase was seen in the low albumin groups (group 5-7) compared to the normal albumin group regarding the rate of all examined systemic and local complications (Fig. 1-2). The lowest measured albumin levels throughout hospitalization $(n=1272)$ were 
significantly and dose-dependently associated with severity $(p<0.001)$, mortality $(p<0.001)$, length of stay $(p<0.001)$, and maximum CRP values $(p<0.001)$ (Fig. 3).

Moderate and severe AP and mortality is associated with significantly lower albumin levels and greater albumin loss

Albumin loss was analyzed using data from patients with at least two albumin measurements $(n=335$; Supplementary Fig. S18). Compared to mild cases, patients with moderate and severe AP showed a greater decrease in albumin levels (medians 5.4 vs. 9 and $15.25 \mathrm{~g} / \mathrm{L} ; \mathrm{p}<0.001$ for both comparisons). The comparison of delta albumin between the moderate and severe groups also yielded significant results $(p=0.003)$. Patients who died also lost significantly more albumin during hospitalization (medians 6.7 vs. $15.75 \mathrm{~g} / \mathrm{L} ; \mathrm{p}=0.002$ ). The median time to the lowest albumin levels from admission was 4 days (IQR: $3-7$ days).

AP patients with less than $25 \mathrm{~g} / \mathrm{L}$ serum albumin have a 16.8 times higher risk of death, and 48.8 times higher risk of severe AP compared to patients with normal albumin levels

Age is an independent risk factor for severe AP and mortality, whereas hypertriglyceridemia-induced and idiopathic AP and the combination of alcoholic and biliary causes are independently associated with mortality (Table 2 and Supplementary Table S2). Hypoalbuminemia below 25-29.99 g/L (OR: 2.912; Cl: 1.176-6.893) and below $25 \mathrm{~g} / \mathrm{L}$ (OR: 16.828; Cl: 8.323-35.129) were associated with an increased risk of mortality (Table 2). In a separate analysis, hypoalbuminemia ( $<35 \mathrm{~g} / \mathrm{L}$ ) was also an independent risk factor for mortality (OR: 4.185; Cl: 2.286-8.039) (Table S3). Furthermore, hypoalbuminemia anytime during hospitalization was associated with a higher risk for severe AP (OR: 10.664; Cl: 6.188-19.614), and a gradual increase of odds ratios can be observed in the low albumin groups (OR: 2.359; Cl: 1.0305.240 for group 5; OR: 11.709; Cl: 6.038-23.515 for group 6 and OR: 48.761; Cl: 25.276-98.908 for group 7). 
Table 2

Logistic regression for severity and mortality using the lowest measured albumin cohort Lowest measured albumin $(n=1272)$ - mortality

\begin{tabular}{|c|c|c|c|c|c|c|}
\hline Predictor & & $\beta$ & SE & OR & $95 \% \mathrm{Cl}$ & $\mathrm{p}$ \\
\hline $\begin{array}{l}\text { On-admission } \\
\text { albumin level }\end{array}$ & $\begin{array}{l}30-34.99 \mathrm{~g} / \mathrm{L}(\mathrm{vs} . \\
\geq 35 \mathrm{~g} / \mathrm{L})\end{array}$ & -0.016 & 0.531 & 0.984 & $\begin{array}{l}0.313- \\
2.621\end{array}$ & 0.976 \\
\hline & $\begin{array}{l}25-29.99 \mathrm{~g} / \mathrm{L} \text { (vs. } \\
\geq 35 \mathrm{~g} / \mathrm{L})\end{array}$ & 1.069 & 0.448 & 2.912 & $\begin{array}{l}1.166- \\
6.893\end{array}$ & 0.017 \\
\hline & $\begin{array}{l}<25 \mathrm{~g} / \mathrm{L} \text { (vs. } \geq 35 \\
\mathrm{~g} / \mathrm{L})\end{array}$ & 2.823 & 0.365 & 16.828 & $\begin{array}{l}8.323- \\
35.129\end{array}$ & $\begin{array}{l}<.001 \\
0.001\end{array}$ \\
\hline Age & per years & 0.043 & 0.012 & 1.044 & $\begin{array}{l}1.021- \\
1.070\end{array}$ & $\hat{0.001}$ \\
\hline Gender & female (vs. male) & -0.352 & 0.347 & 0.703 & $\begin{array}{l}0.352- \\
1.380\end{array}$ & 0.309 \\
\hline Etiology & alcohol (vs. biliary) & 0.909 & 0.523 & 2.481 & $\begin{array}{l}0.880- \\
6.960\end{array}$ & 0.083 \\
\hline & HTG (vs. biliary) & 1.569 & 0.766 & 4.803 & $\begin{array}{l}0.914- \\
19.900\end{array}$ & 0.041 \\
\hline & $\begin{array}{l}\text { biliary + alcohol (vs. } \\
\text { biliary) }\end{array}$ & 1.651 & 0.793 & 5.215 & $\begin{array}{l}0.949- \\
22.798\end{array}$ & 0.037 \\
\hline & $\begin{array}{l}\text { biliary + HTG (vs. } \\
\text { biliary) }\end{array}$ & -12.335 & 786.272 & - & - & 0.987 \\
\hline & $\begin{array}{l}\text { alcohol + HTG (vs. } \\
\text { biliary) }\end{array}$ & 1.356 & 0.793 & 3.880 & $\begin{array}{l}0.709- \\
17.009\end{array}$ & 0.087 \\
\hline & idiopathic (vs. biliary) & 1.402 & 0.402 & 4.063 & $\begin{array}{l}1.878- \\
9.181\end{array}$ & $\begin{array}{l}< \\
0.001\end{array}$ \\
\hline & other (vs. biliary) & 0.213 & 0.807 & 1.237 & $\begin{array}{l}0.182- \\
5.045\end{array}$ & 0.792 \\
\hline Lowest measur & in $(n=1272)$ - severity & & & & & \\
\hline Predictor & & $\beta$ & SE & OR & $95 \% \mathrm{Cl}$ & $\mathrm{p}$ \\
\hline $\begin{array}{l}\text { On-admission } \\
\text { albumin }\end{array}$ & $\begin{array}{l}30-34.99 \mathrm{~g} / \mathrm{L}(\mathrm{v} . \geq 35 \\
\mathrm{g} / \mathrm{L})\end{array}$ & 0.858 & 0.410 & 2.359 & $\begin{array}{l}1.030- \\
5.240\end{array}$ & 0.036 \\
\hline & $\begin{array}{l}25-29.99 \mathrm{~g} / \mathrm{L}(\mathrm{v} . \geq 35 \\
\mathrm{g} / \mathrm{L})\end{array}$ & 2.460 & 0.345 & 11.709 & $\begin{array}{l}6.038- \\
23.515\end{array}$ & $\begin{array}{l}<.001 \\
0.01\end{array}$ \\
\hline & $<25 \mathrm{~g} / \mathrm{L}(\mathrm{v} . \geq 35 \mathrm{~g} / \mathrm{L})$ & 3.887 & 0.346 & 48.761 & $\begin{array}{l}25.276- \\
98.908\end{array}$ & $\begin{array}{l}<.001 \\
0.01\end{array}$ \\
\hline Age & per years & 0.032 & 0.009 & 1.032 & $\begin{array}{l}1.015- \\
1.051\end{array}$ & ¿.001 \\
\hline
\end{tabular}




\begin{tabular}{|c|c|c|c|c|c|c|}
\hline \multicolumn{7}{|c|}{ Lowest measured albumin $(n=1272)$ - mortality } \\
\hline Gender & female (vs. male) & -0.332 & 0.274 & 0.718 & $\begin{array}{l}0.417- \\
1.225\end{array}$ & 0.226 \\
\hline \multirow[t]{7}{*}{ Etiology } & alcohol (vs. biliary) & 0.093 & 0.403 & 1.097 & $\begin{array}{l}0.492- \\
2.403\end{array}$ & 0.818 \\
\hline & HTG (vs. biliary) & 1.060 & 0.565 & 2.885 & $\begin{array}{l}0.910- \\
8.476\end{array}$ & 0.061 \\
\hline & $\begin{array}{l}\text { biliary + alcohol (vs. } \\
\text { biliary) }\end{array}$ & 0.172 & 0.778 & 1.188 & $\begin{array}{l}0.222- \\
5.006\end{array}$ & 0.825 \\
\hline & $\begin{array}{l}\text { biliary + HTG (vs. } \\
\text { biliary) }\end{array}$ & -13.429 & 753.256 & - & - & 0.986 \\
\hline & $\begin{array}{l}\text { alcohol + HTG (vs. } \\
\text { biliary) }\end{array}$ & 0.497 & 0.657 & 1.643 & $\begin{array}{l}0.422- \\
5.688\end{array}$ & 0.450 \\
\hline & idiopathic (vs. biliary) & 0.541 & 0.320 & 1.718 & $\begin{array}{l}0.915- \\
3.218\end{array}$ & 0.091 \\
\hline & other (vs. biliary) & 0.008 & 0.547 & 1.008 & $\begin{array}{l}0.310- \\
2.744\end{array}$ & 0.988 \\
\hline
\end{tabular}

HTG: hypertriglyceridemia; $\beta$ : $\beta$ coefficient; SE: standard error; OR: odds ratio; Cl: confidence interval

The lowest albumin values have good and fair predictive value for severity and mortality in acute pancreatitis

The lowest measured albumin levels have higher AUC values: 0.848 for severity and 0.747 for mortality (Fig. 3). The best cut-off values were $31.3 \mathrm{~g} / \mathrm{L}$ for severity (sensitivity: $82.9 \%$, specificity: $76.4 \%$ ) and 28.6 $\mathrm{g} / \mathrm{L}$ for mortality (sensitivity: $89.9 \%$, specificity: $56.1 \%$ ). The day of the lowest albumin measurement ranged from 1 to 56 days, with a median of 2 days. Most patients only had a single measurement around the time of admission.

\section{Discussion}

To date, this is the most comprehensive evaluation of AP patients with hypoalbuminemia, using the largest, prospectively collected, high-quality dataset.

We found that almost one-fifth of patients had hypoalbuminemia on admission (19\%), and a further $25 \%$ developed hypoalbuminemia during hospitalization, meaning that every third patient was affected.

In our analysis, hypoalbuminemia under $25 \mathrm{~g} / \mathrm{L}$ anytime during hospitalization was independently associated with a more than 47 times higher chance for severe AP and a more than 16 times higher chance for mortality. 
Our findings are in line with results regarding hypoalbuminemia in other diseases. Hypoalbuminemia was a prominent risk factor in community-acquired bloodstream infection with severe sepsis and septic shock 8. A retrospective analysis from more than 20.000 emergency medical patients' data from Ireland revealed that hypoalbuminemia is independently associated with 30-day in-hospital mortality, with a nonlinear relationship between mortality and on-admission albumin levels ${ }^{9}$. Moreover, in a secondary analysis of a prospective cohort, AP patients with multiorgan failure (MOF; $n=18$ ) demonstrated a sharper decline in serum albumin $(P<0.001)$ compared to non-MOF patients $(n=39)^{10}$.

We did not only prove that hypoalbuminemia is a risk factor but showed the dose-dependent association between low albumin levels and severity, mortality, number of patients with any local complications, number of patients developing organ failure, and maximum CRP levels in both analyses (on-admission and lowest measured albumin levels).

These associations can be explained by the numerous physiological functions of human serum albumin. For long, albumin was considered a negative acute-phase protein, with decreasing production giving way to inflammatory cytokines in inflammation ${ }^{11}$. Serum albumin levels undoubtedly decrease in inflammatory states, which may be due to a shorter half-life and a larger interstitial pool which causes the dilution of albumin ${ }^{12-14}$. Capillary leak consequential to inflammatory processes plays a role in the decline of serum albumin, but it is argued that the escape of albumin to the tissues may be beneficial because of its antioxidant and scavenging activity ${ }^{15}$. However, a more than two times higher production rate was observed in critically ill ICU patients; this increased production can still not balance the higher demand. This can be considered as a relative synthetic insufficiency of hepatic function ${ }^{16}$.

In our analysis, albumin loss was significantly associated with severity and mortality. However, only $51.7 \%$ of patients had albumin measurements at least once, and $13.6 \%$ at least twice during their hospitalization in the HPSG database. This highlights how neglected albumin measurements are in AP.

From the clinician's point of view, the decline of serum albumin levels - regardless of on-admission albumin levels - signals clinical worsening and may help identify high-risk AP patients. However, clinicians mostly miss the opportunity to pre-emptively and frequently measure serum albumin, delaying timely intervention.

To date, no clinical trial examined therapeutic albumin administration in AP. As we know, albumin is similarly associated with outcomes in sepsis and septic shock; randomized controlled trials on this field could be a starting block ${ }^{15,17}$. The controversial results of studies and meta-analyses on this field may be explained by heterogeneous patient populations and the time sensitivity of this treatment ${ }^{18}$.

To exploit the potential in therapeutic albumin administration in AP, further, more detailed clinical studies are needed to identify the patient subpopulations benefiting the most from this therapeutical option.

\section{Conclusion}


Hypoalbuminemia is remarkably common in AP (seen in 19\% of patients on-admission and $35.7 \%$ during hospitalization) and an independent risk factor of severity, mortality. Importantly, albumin loss during hospitalization was also associated with severity and mortality, suggesting that routine monitoring of serum albumin is recommended, and albumin administration should be examined as a therapeutic intervention in AP.

Implications for research: Clinical trials assessing the potential benefit of albumin replacement in AP are needed.

Implication for practice: 1) Albumin levels should be measured for all AP patients, 2) Albumin levels should be controlled at least in those patients whose condition is worsening during AP, 3) Albumin administration should be considered at least in those patients with severe hypoalbuminemia $(<25 \mathrm{~g} / \mathrm{L})$.

\section{Methods}

\section{Study design and definitions}

This analysis of an international, prospective, multicenter cohort was done using data from the Acute Pancreatitis Registry of the Hungarian Pancreatic Study Group (HPSG) ${ }^{19}$. Patient data were collected from registry establishment until 31 December 2019 on electronic case report forms and validated using a four-tiered data validation protocol. Contributing centers are shown in the supplementary material (Table and Fig. S1). The registry was approved by the Scientific and Research Ethics Committee of the Medical Research Council (222254-1/2012/EKU) in 2012. It conforms to the Declaration of Helsinki revised in 2013. All participants provided written informed consent. Data collection and validation are detailed by Párniczky et al. ${ }^{20}$. The Hungarian Pancreatic Study Group published analyses from the registry, the population of which may overlap with our analyzed cohort ${ }^{20-30}$.

Diagnosis of AP was established using the IAP/APA guidelines ${ }^{31}$, while severity and complications were defined using the Revised Atlanta Classification ${ }^{1}$.

\section{Participants}

To answer a post-hoc clinical research question, analyses were performed on patients' data with albumin measurement anytime during hospitalization (lowest measured albumin cohort, $n=1272$ ) and in the first 48 hours of hospitalization (on-admission albumin cohort, $n=1149$ ). The cut-off value between the low and normal albumin group was $35 \mathrm{~g} / \mathrm{L}$ in both cases, based on the commonly used lower normal value. Subjects were further divided into 7 subgroups (group 1 to 7$)$ using the lowest $(n=1272)$ or first measured $(n=1149)$ albumin values.

In the analyses of albumin change, selected patients $(n=335)$ with at least two albumin measurements were included. Delta albumin was calculated as the difference between the first and lowest measured albumin levels. 


\section{Statistical analysis}

Descriptive statistics are presented as median with $25 \%$ and $75 \%$ percentiles (IQR) or mean with standard deviation (SD) for continuous variables and as numbers and proportions for categorical variables.

Chi-squared test or Fisher's exact test were used for the assessment of the relationship between categorical variables. Mann-Whitney U test or Kruskal-Wallis test followed by Dunnett's post hoc test was used to evaluate differences between groups in case of continuous variables.

Multivariate binary logistic regression analysis was performed to identify the risk factors independently associated with severe disease and mortality. Odds ratios (OR) with $95 \%$ confidence intervals (Cl) were calculated.

Receiver Operator Characteristic (ROC) curve and Area Under the Curve (AUC) with $95 \% \mathrm{Cl}$ was used to identify the ability of albumin levels predicting mortality or severity of AP (AUC between $0.5-0.6$ was considered as fail, between $0.6-0.7$ as poor, between $0.7-0.8$ as fair, between $0.8-0.9$ as good and above 0.9 as excellent). Best cut-offs were calculated by using the Youden index ${ }^{32}$.

$\mathrm{P}<0.05$ was considered statistically significant, except for the Kruskal-Wallis test followed by Dunnett's post hoc test, where $p<0.025$ was considered statistically significant.

All analyses were carried out in R statistical software, version 4.0.2 (R Core Team, 2020, Vienna, Austria), packages: pROC (v. 1.17.0.1) and PMCMRplus (v. 1.9.0.) ${ }^{33,34}$.

\section{Representativity}

The main characteristics of the analyzed cohorts are in accordance with literature data. However, they differed significantly from the entire cohort $(n=2461)$ in terms of severity, length of stay, and mortality (Fig. S2).

\section{Reporting}

We report our results following The Strengthening the Reporting of Observational Studies in Epidemiology (STROBE) Statement, using the provided checklist ${ }^{35}$.

\section{Data availability}

The full dataset is available upon reasonable request.

\section{Strengths And Limitations}

We conducted the most extensive, most comprehensive cohort study on the role of hypoalbuminemia in acute pancreatitis to date. We analyzed high-quality data from a prospective, international, multicentric registry. We identified hypoalbuminemia as an independent risk factor in AP, present in at least every third 
patient. We also found a dose-dependent relationship between albumin levels and main outcomes, which was previously not described.

Among the limitations, we must mention the arbitrary classification of albumin levels (except for the lownormal cut-off), the missing data on albumin levels and albumin administration during the hospital stay, and the limited number of albumin measurements during the hospital stay, which could introduce bias. Our analyzed cohorts differed from the total cohort in some aspects, which may signal performance bias, as albumin measurements are more frequently ordered for patients with expected hypoalbuminemia.

\section{Declarations}

\section{Acknowledgments}

We would like to thank all patients and personnel who contributed to the registry and this analysis. The authors are very thankful to those collaborators who uploaded clinical data but did not reach the necessary contribution to be among the authors.

Our study was supported by the Human Resources Development Operational Programme Grant "EFOP3.6.2-16-2017-00006 - LIVE LONGER" and the Economic Development and Innovation Operative Programme Grant "GINOP-2.3.2-15-2016-00015 - I-KOM" co-financed by the European Union (European Regional Development Fund) within the framework of Programme Széchenyi 2020 (PH) co-financed by the European Union. Further grants: János Bolyai Research Scholarship from the Hungarian Academy of

Sciences and ÚNKP-20-5 New National Excellence Program of the Ministry for Innovation and Technology from the source of the National Research, Development and Innovation Fund (AP and AM) and National Research, Development and Innovation Office Project Grant (K116634, PH, FK131864, AM). The funders played no role in planning the concept, data analysis, interpretation, writing of the manuscript and choosing to publish.

\section{Author contributions}

$\mathrm{KO}, \mathrm{PH}$ and $\mathrm{AP}$ drafted the concept and interpreted the data. KO wrote the majority of the manuscript. DN and LS performed the statistical analyses. VZ prepared the figures. JB, SG, PS, LC, FI, JH, MP, MV, IT, AM, VS, ERM, SG, PK, RH, BE and ZM all provided a substantial number of enrolled participants. AM, ZS, MI and PJH performed patient enrollment and supervised data quality. NF and OF acted as a radiological supervisor, ensuring data quality. AM and TN provided insight on laboratory markers and contributed to patient enrollment. AS, PH and AP provided methodological and medical guidance and supervised the writing of the article. All co-authors have read and approved the final version of the manuscript.

The authors declare no conflict of interest.

\section{Data availability}

The full dataset is available upon reasonable request. 


\section{References}

1 Sarr, M. G. 2012 revision of the Atlanta classification of acute pancreatitis. Polskie Archiwum Medycyny Wewnetrznej 123, 118-124, doi:10.20452/pamw.1627 (2013).

2 Oettl, K. \& Stauber, R. E. Physiological and pathological changes in the redox state of human serum albumin critically influence its binding properties. British journal of pharmacology 151, 580-590, doi:10.1038/sj.bjp.0707251 (2007).

3 Stamler, J. S. et al. Nitric oxide circulates in mammalian plasma primarily as an S-nitroso adduct of serum albumin. Proceedings of the National Academy of Sciences of the United States of America 89, 7674-7677, doi:10.1073/pnas.89.16.7674 (1992).

$4 \quad$ Ulldemolins, M., Roberts, J. A., Rello, J., Paterson, D. L. \& Lipman, J. The effects of hypoalbuminaemia on optimizing antibacterial dosing in critically ill patients. Clinical pharmacokinetics 50, 99-110, doi:10.2165/11539220-000000000-00000 (2011).

5 Zhang, W. et al. Evaluation of Early Prognostic Factors of Mortality in Patients with Acute Pancreatitis: A Retrospective Study. Gastroenterology research and practice 2017, 8363561, doi:10.1155/2017/8363561 (2017).

6 Wang, X. et al. Nosocomial mortality and early prediction of patients with severe acute pancreatitis. Journal of gastroenterology and hepatology 25, 1386-1393, doi:10.1111/j.14401746.2010.06376.x (2010).

$7 \quad$ Hong, W. et al. Serum Albumin Is Independently Associated with Persistent Organ Failure in Acute Pancreatitis. Canadian journal of gastroenterology \& hepatology 2017, 5297143, doi:10.1155/2017/5297143 (2017).

8 Artero, A. et al. Prognostic factors of mortality in patients with community-acquired bloodstream infection with severe sepsis and septic shock. Journal of critical care 25, 276-281, doi:10.1016/j.jcrc.2009.12.004 (2010).

9 Lyons, O., Whelan, B., Bennett, K., O'Riordan, D. \& Silke, B. Serum albumin as an outcome predictor in hospital emergency medical admissions. European journal of internal medicine 21, 17-20, doi:10.1016/j.ejim.2009.10.010 (2010).

10 Komara, N. L. et al. Severe acute pancreatitis: capillary permeability model linking systemic inflammation to multiorgan failure. American journal of physiology. Gastrointestinal and liver physiology 319, G573-g583, doi:10.1152/ajpgi.00285.2020 (2020).

11 Charlie-Silva, I. et al. Acute-phase proteins during inflammatory reaction by bacterial infection: Fish-model. Scientific Reports 9, 4776, doi:10.1038/s41598-019-41312-z (2019). 
12 Barle, H. et al. Growth hormone does not affect albumin synthesis in the critically ill. Intensive Care Medicine 27, 836-843, doi:10.1007/s001340100914 (2001).

13 Barle, H. et al. Synthesis rates of total liver protein and albumin are both increased in patients with an acute inflammatory response. Clinical Science 110, 93-99, doi:10.1042/CS20050222 (2005).

14 Dahn, M. S., Mitchell, R. A., Lange, M. P., Smith, S. \& Jacobs, L. A. Hepatic metabolic response to injury and sepsis. Surgery 117, 520-530, doi:10.1016/s0039-6060(05)80251-x (1995).

15 Soeters, P. B., Wolfe, R. R. \& Shenkin, A. Hypoalbuminemia: Pathogenesis and Clinical Significance. 43, 181-193, doi:https://doi.org/10.1002/jpen.1451 (2019).

16 Levitt, D. G. \& Levitt, M. D. Human serum albumin homeostasis: a new look at the roles of synthesis, catabolism, renal and gastrointestinal excretion, and the clinical value of serum albumin measurements. Int J Gen Med 9, 229-255, doi:10.2147/ijgm.S102819 (2016).

17 Vincent, J.-L. et al. Albumin administration in the acutely ill: what is new and where next? Critical Care 18, 231, doi:10.1186/cc13991 (2014).

18 Flannery, A. H., Owen, G. D., Coz, A., Thompson Bastin, M. L. \& Patel, K. Impact of Hyperoncotic Albumin on Duration of Vasopressor Support inSeptic Shock: A PropensityScore-Matched Analysis. The Annals of pharmacotherapy, 1060028020963645, doi:10.1177/1060028020963645 (2020).

19 Acute Pancreatitis Registry.

20 Párniczky, A. et al. Antibiotic therapy in acute pancreatitis: From global overuse to evidence based recommendations. Pancreatology : official journal of the International Association of Pancreatology (IAP) ... [et al.] 19, 488-499, doi:10.1016/j.pan.2019.04.003 (2019).

21 Párniczky, A. et al. Prospective, Multicentre, Nationwide Clinical Data from 600 Cases of Acute Pancreatitis. PloS one 11, e0165309, doi:10.1371/journal.pone.0165309 (2016).

22 Mosztbacher, D. et al. Hypertriglyceridemia-induced acute pancreatitis: A prospective, multicenter, international cohort analysis of 716 acute pancreatitis cases. Pancreatology : official journal of the International Association of Pancreatology (IAP) ... [et al.] 20, 608-616, doi:10.1016/j.pan.2020.03.018 (2020).

23 Farkas, N. et al. A Multicenter, International Cohort Analysis of 1435 Cases to Support Clinical Trial Design in Acute Pancreatitis. Frontiers in physiology 10, 1092, doi:10.3389/fphys.2019.01092 (2019).

24 Hágendorn, R. et al. Development of disturbance of consciousness is associated with increased severity in acute pancreatitis. Pancreatology : official journal of the International Association of Pancreatology (IAP) ... [et al.] 20, 806-812, doi:10.1016/j.pan.2020.05.009 (2020). 
25 Demcsák, A. et al. Acid suppression therapy, gastrointestinal bleeding and infection in acute pancreatitis - An international cohort study. Pancreatology : official journal of the International Association of Pancreatology (IAP) ... [et al.] 20, 1323-1331, doi:10.1016/j.pan.2020.08.009 (2020).

26 Gódi, S. et al. Centralized care for acute pancreatitis significantly improves outcomes. Journal of gastrointestinal and liver diseases : JGLD 27, 151-157, doi:10.15403/jgld.2014.1121.272.pan (2018).

27 Szakács, Z. et al. Aging and Comorbidities in Acute Pancreatitis II.: A Cohort-Analysis of 1203 Prospectively Collected Cases. Frontiers in physiology 9, 1776, doi:10.3389/fphys.2018.01776 (2018).

28 Halász, A. et al. Outcomes and timing of endoscopic retrograde cholangiopancreatography for acute biliary pancreatitis. Digestive and liver disease : official journal of the Italian Society of Gastroenterology and the Italian Association for the Study of the Liver 51, 1281-1286, doi:10.1016/j.dld.2019.03.018 (2019).

29 Szentesi, A. et al. Multiple Hits in Acute Pancreatitis: Components of Metabolic Syndrome Synergize Each Other's Deteriorating Effects. Frontiers in physiology 10, 1202, doi:10.3389/fphys.2019.01202 (2019).

30 Hegyi, P. J. et al. Evidence for diagnosis of early chronic pancreatitis after three episodes of acute pancreatitis: a cross-sectional multicentre international study with experimental animal model. Scientific Reports 11, 1367, doi:10.1038/s41598-020-80532-6 (2021).

31 IAP/APA evidence-based guidelines for the management of acute pancreatitis. Pancreatology : official journal of the International Association of Pancreatology (IAP) ... [et al.] 13, e1-e15, doi:https://doi.org/10.1016/j.pan.2013.07.063 (2013).

32 Fluss, R., Faraggi, D. \& Reiser, B. Estimation of the Youden Index and its associated cutoff point. Biom J 47, 458-472, doi:10.1002/bimj.200410135 (2005).

33 Robin, X. et al. pROC: an open-source package for R and S+ to analyze and compare ROC curves. BMC Bioinformatics 12, 77, doi:10.1186/1471-2105-12-77 (2011).

34 PMCMRplus: Calculate Pairwise Multiple Comparisons of Mean Rank Sums Extended.

35 von Elm, E. et al. The Strengthening the Reporting of Observational Studies in Epidemiology (STROBE) statement: guidelines for reporting observational studies. Lancet (London, England) 370, 14531457, doi:10.1016/s0140-6736(07)61602-x (2007).

\section{Figures}




\begin{tabular}{|c|c|c|c|c|c|c|}
\hline \multicolumn{7}{|c|}{ GROUP: albumin $(\mathrm{g} / \mathrm{L})$} \\
\hline \multicolumn{4}{|c|}{ NORMAL } & \multicolumn{3}{|c|}{ Low } \\
\hline $1:>50$ & $2: 49.9-45$ & 3: $44.99-40$ & 4: $39.99-35$ & $5: 34.99-30$ & $6: 29.9-25$ & $7:<24.99$ \\
\hline
\end{tabular}

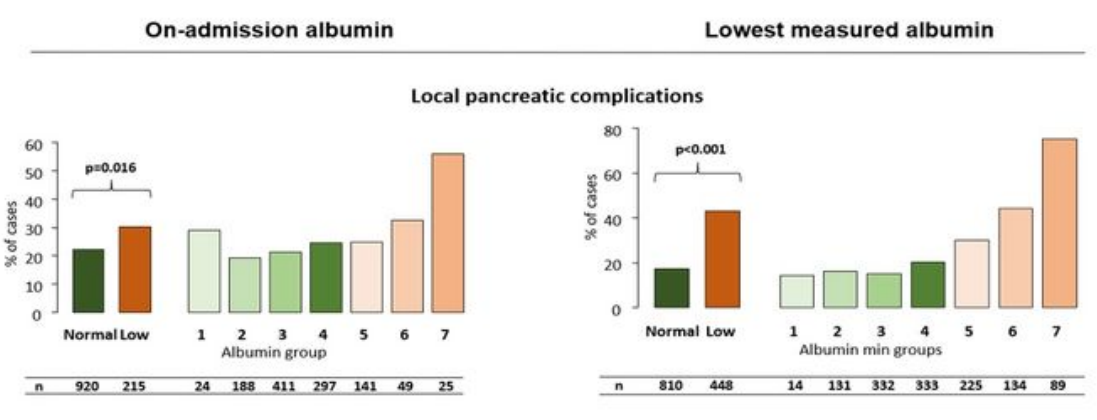

Pancreatic pseudocyst
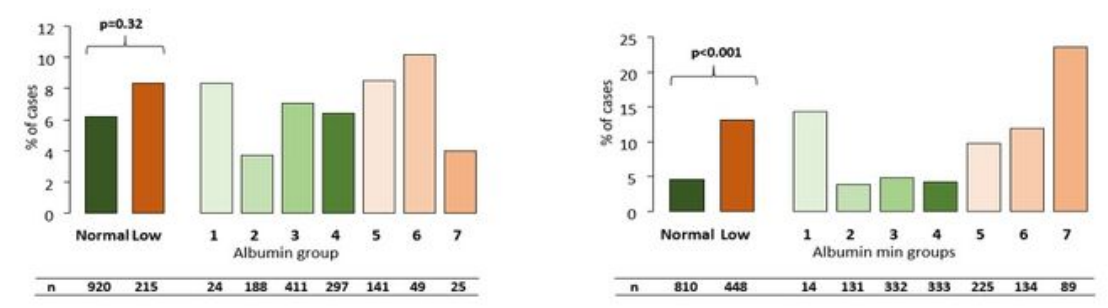

Pancreatic necrosis
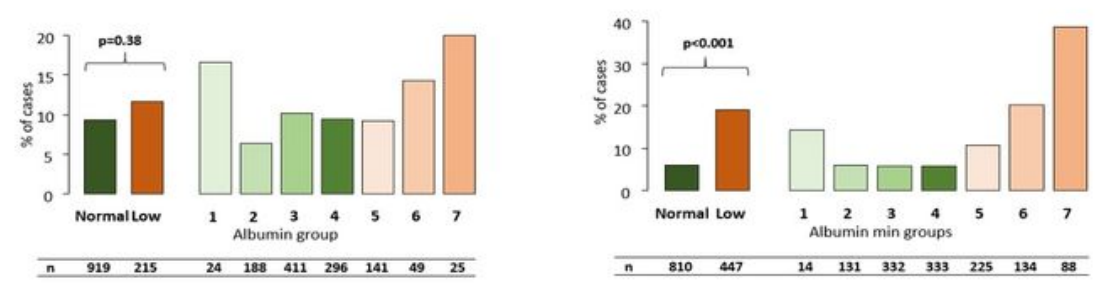

Peripancreatic fluid collection
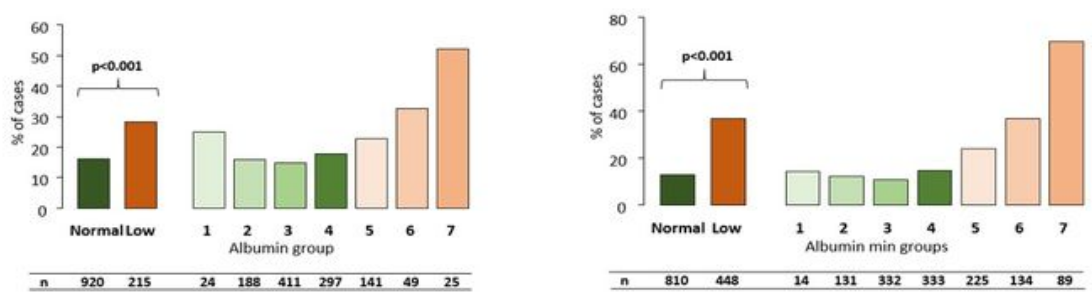

\section{Figure 1}

Association between albumin level and local complications defined by the Revised Atlanta Criteria in acute pancreatitis All types of local complications were significantly more frequent in the low albumin group. A dose-dependent increase was seen in the rate of local complications and peripancreatic fluid collection in both cohorts and in pancreatic necrosis and pseudocyst in the lowest measured albumin 
cohort. $\mathrm{P}<0.05$ is considered significant. Patients with albumin levels $<35 \mathrm{~g} / \mathrm{L}$ were included in the low albumin group (groups 5-7).

\begin{tabular}{|c|c|c|c|c|c|c|}
\hline \multicolumn{7}{|c|}{ GROUP: albumin $(\mathrm{g} / \mathrm{L})$} \\
\hline \multicolumn{4}{|c|}{ NORMAL } & \multicolumn{3}{|c|}{ Low } \\
\hline $1:>50$ & $2: 49.9-45$ & 3: $44.99-40$ & $4: 39.99-35$ & 5: $34.99-30$ & $6: 29.9-25$ & $7:<24.99$ \\
\hline
\end{tabular}

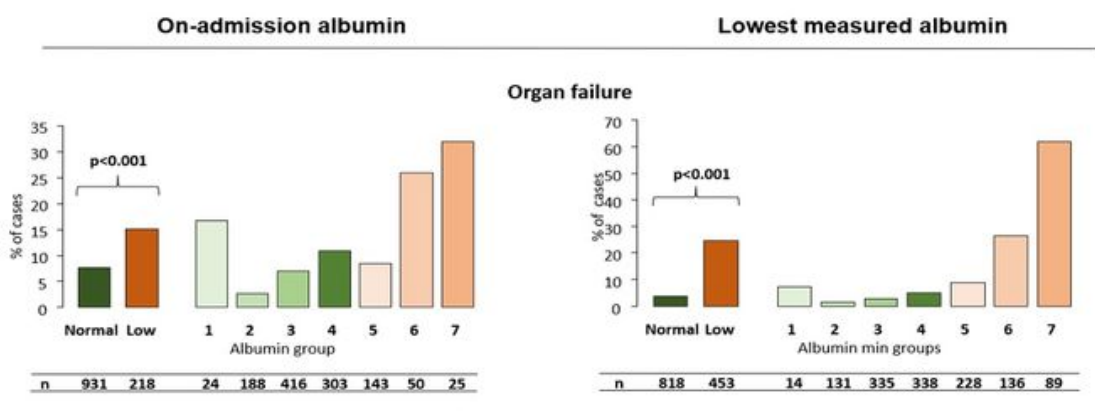

Respiratory failure
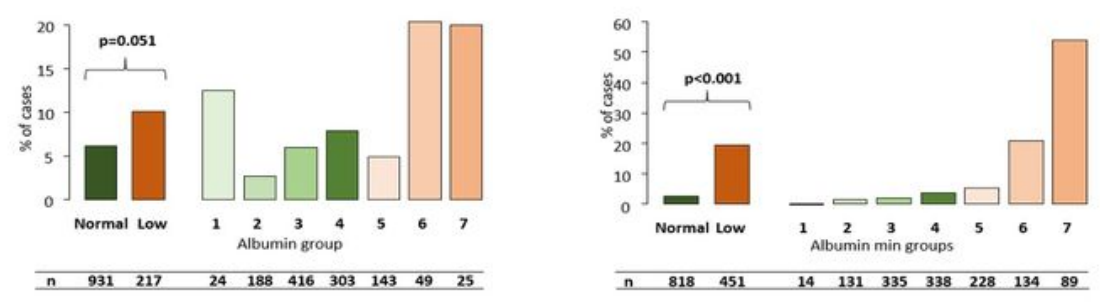

Heart failure
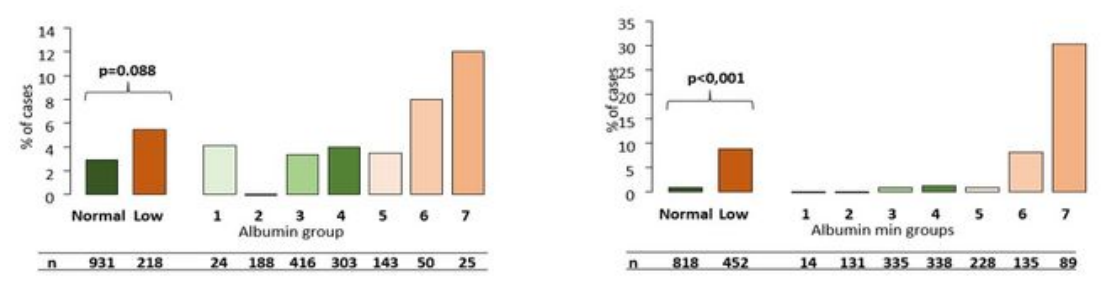

Renal failure
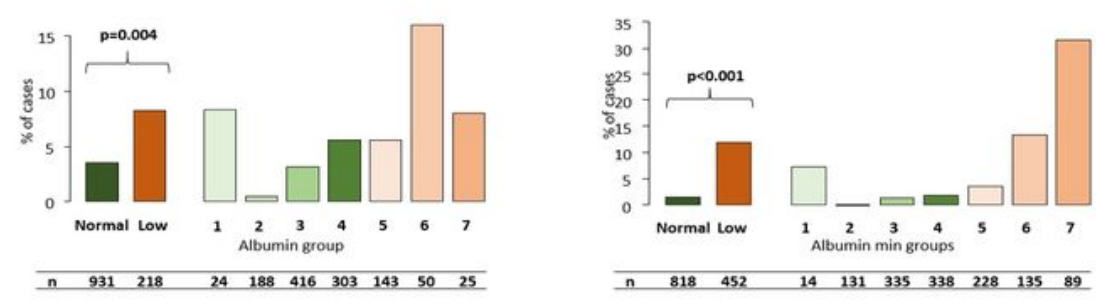

\section{Figure 2}

Association between albumin level and organ failures, defined by the Revised Atlanta Criteria in acute pancreatitis Significantly more patients developed organ failure in the low albumin group in both cohorts. A dose-dependent increase was seen in the case of all analyses in the lowest measured albumin cohort. 
Heart failure was dose-dependently increased in the on-admission cohort as well. $\mathrm{P}<0.005$ is considered significant.

\begin{tabular}{|c|c|c|c|c|c|c|}
\hline \multicolumn{7}{|c|}{ GROUP: albumin (g/L) } \\
\hline $1:>50$ & $2: 49.9-45$ & $3: 44.99-40$ & $4: 39.99-35$ & $5: 34.99-30$ & $6: 29.9-25$ & $7:<24.99$ \\
\hline
\end{tabular}

\begin{tabular}{cc} 
On-admission albumin & Lowest measured albumin \\
\hline & Severity \\
"Mild " Moderate * Severe
\end{tabular}
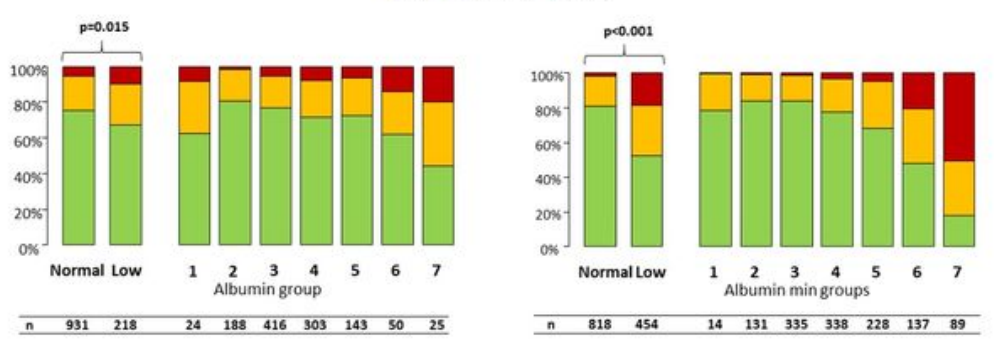

Mortality
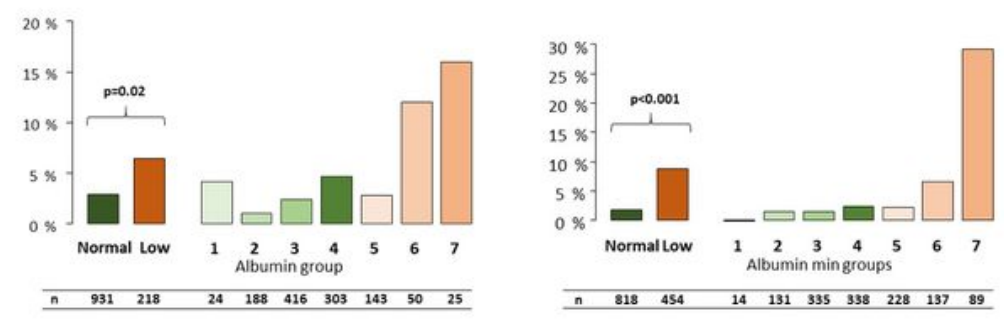

\begin{tabular}{|c|cc|}
\hline Group & $\begin{array}{c}\text { Length of stay } \\
\text { Days (mean, SD) }\end{array}$ & $\begin{array}{c}\text { Mortality } \\
\text { event n }(\%)\end{array}$ \\
\hline $\mathbf{1}$ & $8.92,5.81$ & $1(4.17)$ \\
2 & $8.19,7.29$ & $2(1.06)$ \\
$\mathbf{3}$ & $\mathbf{8 . 8 6 , 1 0 . 0 2}$ & $10(2.40)$ \\
$\mathbf{4}$ & $9.47,8.44$ & $14(4.62)$ \\
\hline NORMAL & $\mathbf{8 . 9 2 , 8 . 9 3}$ & $\mathbf{2 7}(\mathbf{2 . 9 0 )}$ \\
\hline $\mathbf{5}$ & $9.67,11.35$ & $4(2.80)$ \\
$\mathbf{6}$ & $13.80,18.40$ & $6(12)$ \\
$\mathbf{7}$ & $10.52,5.95$ & $4(16)$ \\
\hline LOW & $\mathbf{1 0 . 7 2 , 1 2 . 9 5}$ & $\mathbf{1 4}(6.42)$ \\
\hline TOTAL & $\mathbf{9 . 2 6 , 9 , 8 4}$ & $\mathbf{4 1 ( 3 . 5 7 )}$ \\
\hline
\end{tabular}

\begin{tabular}{|c|cc|}
\hline Group & $\begin{array}{c}\text { Length of stay } \\
\text { Days (mean, SD) }\end{array}$ & $\begin{array}{c}\text { Mortality } \\
\text { event n }(\%)\end{array}$ \\
\hline $\mathbf{1}$ & $6,2.48$ & $0(0.00)$ \\
$\mathbf{2}$ & $7.11,3.07$ & $2(1.53)$ \\
$\mathbf{3}$ & $7.05,4.42$ & $5(1.49)$ \\
$\mathbf{4}$ & $8.06,4.81$ & $8(2.37)$ \\
\hline NORMAL & $\mathbf{7 . 4 6 , 4 . 4 1}$ & $\mathbf{1 5 ( 1 . 8 3 )}$ \\
\hline $\mathbf{5}$ & $9.78,6.61$ & $5(2.19)$ \\
$\mathbf{6}$ & $15.1,14.6$ & $9(6.47)$ \\
$\mathbf{7}$ & $27.3,25.3$ & $26(29.21)$ \\
\hline LOW & $\mathbf{1 4 . 8 , 1 5 . 9}$ & $\mathbf{4 0}(\mathbf{8 . 8 1})$ \\
\hline TOTAL & $\mathbf{1 0 . 1 , 1 0 . 7 3}$ & $\mathbf{5 5 ( 4 . 3 2 )}$ \\
\hline
\end{tabular}

Maximum C-reactive protein
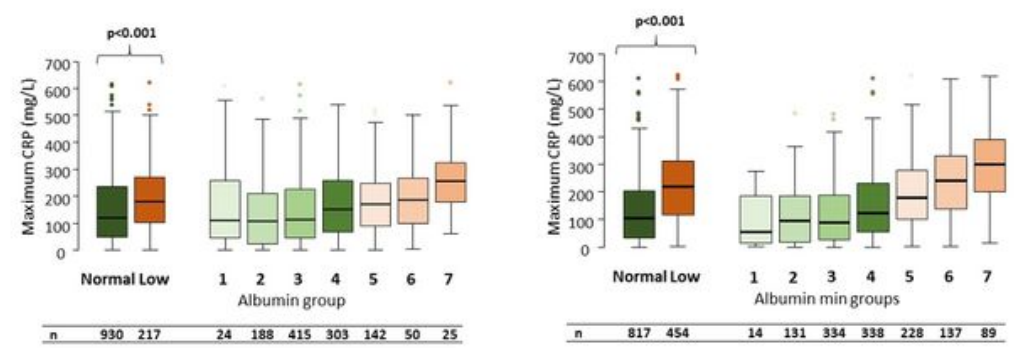

\section{Figure 3}

Association between albumin level and disease severity, mortality, length of stay and maximum Creactive protein level in acute pancreatitis Severity, mortality, length of stay and maximum C-reactive 
protein levels were significantly and dose-dependently associated with hypoalbuminemia in both cohorts. $\mathrm{P}<0.05$ is considered significant.

On-admission albumin

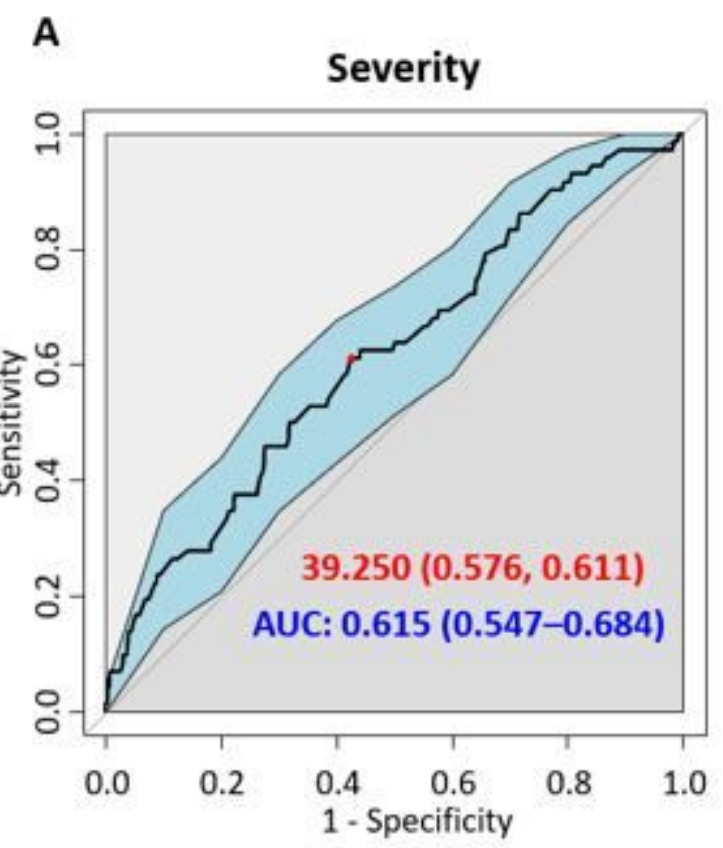

B

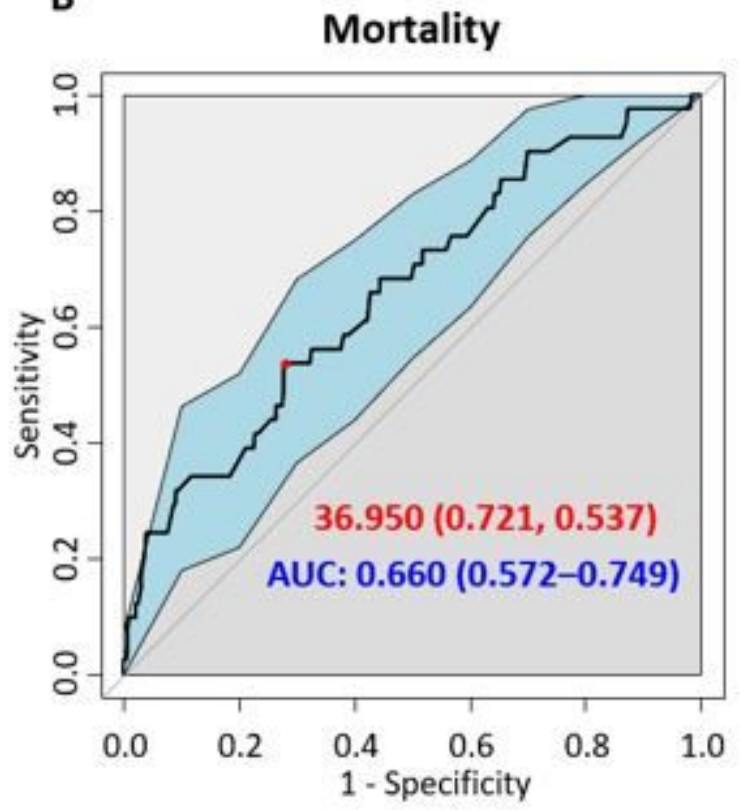

Lowest albumin

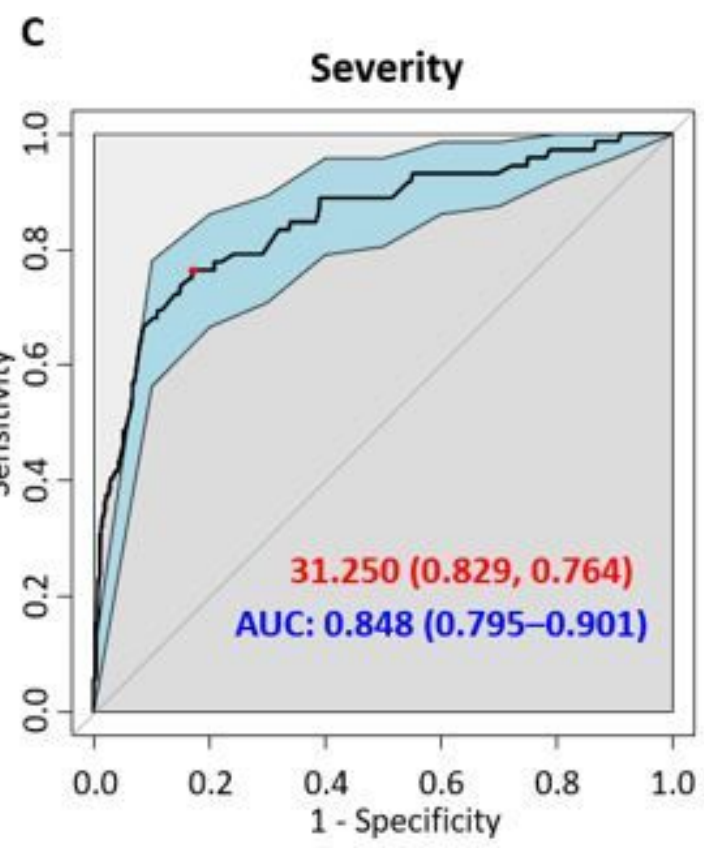

D

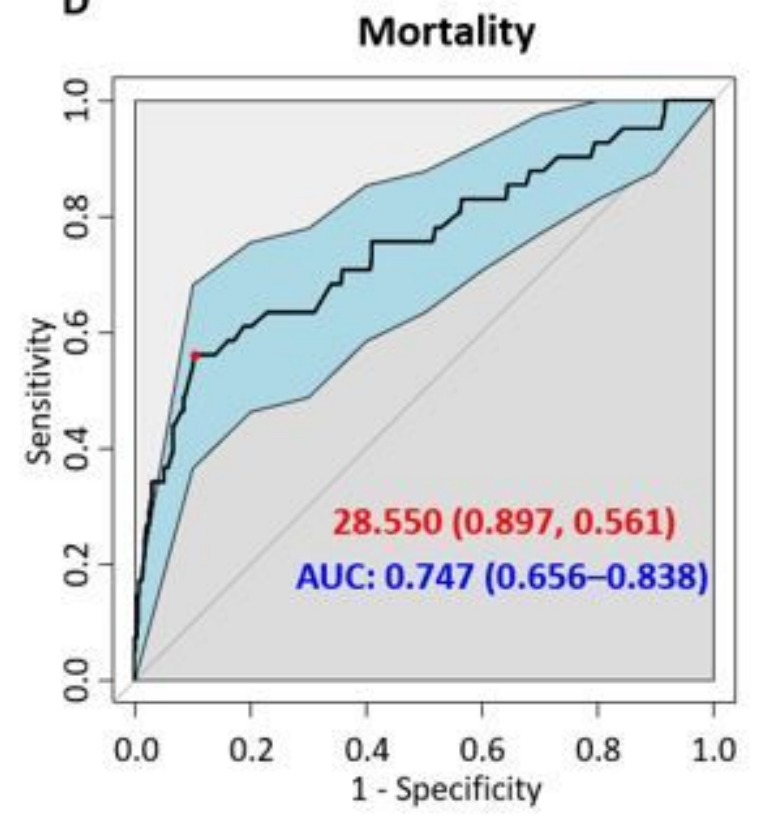

\section{Figure 4}

Receiver operating curves for mortality and severity AUC: area under the curve; best cut-offs are shown in red

\section{Supplementary Files}


This is a list of supplementary files associated with this preprint. Click to download.

- Supplementaryfinal.pdf 